\title{
Smoothing effect of absorption for degenerate parabolic equations in 1-d
}

\author{
Alkis S. Tersenov ${ }^{1}$
}

Received: 14 November 2017 / Accepted: 5 May 2018 / Published online: 24 May 2018

(C) Fondazione Annali di Matematica Pura ed Applicata and Springer-Verlag GmbH Germany, part of Springer Nature 2018

Abstract In the present paper we consider the Cauchy and the Dirichlet problems for the equation

$$
u_{t}=\left(\kappa(t, u) u_{x}\right)_{x}-f(t, u),
$$

where $\kappa(t, u) \geq 0$ for $u \geq 0$. We formulate conditions on $\kappa(t, u)$ and $f(t, u)$ guaranteeing the existence of a global and local solutions such that $u_{x} \in L^{\infty}$ and $u_{t} \in L^{2}$.

Keywords Porous medium equation · Gradient estimates · Quasilinear equations

Mathematics Subject Classification 35K65 -35K59 - 35K20

\section{Introduction and formulation of the results}

Consider the equation

$$
u_{t}=\left(\kappa(t, u) u_{x}\right)_{x}-f(t, u), \quad(t, x) \in \Pi_{T}=(0, T) \times \mathbf{R},
$$

coupled with initial condition

$$
u(0, x)=u_{0}(x) \text { for } x \in \mathbf{R},
$$

where $T>0$ is an arbitrary constant. We assume that

$$
0 \leq u_{0}(x) \leq M, \quad\left|u_{0}(x)-u_{0}(y)\right| \leq K|x-y|, \quad \forall x, y \in \mathbf{R} \quad \text { and } \quad\left\|u_{0 x}\right\|_{L^{2}(\mathbf{R})}<\infty
$$

Alkis S. Tersenov tersenov@uoc.gr

1 Department of Mathematics and Applied Mathematics, University of Crete, 70013 Heraklion, Crete, Greece 
where $M$ and $K$ are some positive constants. Concerning the smoothness of coefficients we suppose that

$$
\kappa(t, u) \in \mathcal{C}_{t, u}^{1,1+\alpha}([0, T] \times(0,+\infty)), \quad f(t, u) \in \mathcal{C}^{\alpha}([0, T] \times(0,+\infty))
$$

for some $\alpha \in(0,1)$. We impose the following structure restrictions

$$
\kappa(t, 0)=f(t, 0)=0, \quad \kappa(t, u) \geq 0 \quad \text { and } \quad f(t, u) \geq 0 \quad \text { for } u \geq 0
$$

and

$$
K^{2}\left[\kappa_{u}\left(t, u_{2}\right)-\kappa_{u}\left(t, u_{1}\right)\right] \leq f\left(t, u_{2}\right)-f\left(t, u_{1}\right) \quad \text { for } u_{2}>u_{1}>0, \quad t \in[0, T] .
$$

Definition 1 We say that a bounded nonnegative Hölder continuous function $u(t, x)$ is a strong solution of problem (1.1), (1.2) if $u_{x} \in L^{\infty}\left(\Pi_{T}\right), u_{t} \in L^{2}\left(\Pi_{T}\right), \sqrt{\kappa(t, u)} u_{x} \in$ $L^{2}\left(\Pi_{T}\right)$, and for an arbitrary smooth $\phi(t, x)$ vanishing for large $|x|$ the following identity is satisfied

$$
\int_{\Pi_{T}} u_{t} \phi \mathrm{d} t \mathrm{~d} x+\int_{\Pi_{T}} \kappa(t, u) u_{x} \phi_{x} \mathrm{~d} t \mathrm{~d} x+\int_{\Pi_{T}} f(t, u) \phi \mathrm{d} t \mathrm{~d} x=0 .
$$

Theorem 1 (global existence) Assume that conditions (1.3)-(1.6) are fulfilled, then for any $T>0$ there exists a strong solution $u(t, x)$ of problem (1.1), (1.2). Moreover

$$
0 \leq u \leq M \quad \forall(t, x) \in \Pi_{T}, \quad\left\|u_{x}\right\|_{L^{\infty}\left(\Pi_{T}\right)} \leq K,
$$

and

$$
\|u\|_{L_{2}(\mathbf{R})}^{2}+2\left\|\sqrt{\kappa} u_{x}\right\|_{L_{2}\left(\Pi_{T}\right)}^{2} \leq\left\|u_{0}\right\|_{L_{2}(\mathbf{R})}^{2} \forall t \in(0, T) .
$$

Let us apply this Theorem to the porous media equation with absorption:

$$
u_{t}=\left(m u^{m-1} u_{x}\right)_{x}-f(t, u) .
$$

Condition (1.6) takes the form

$$
m(m-1) K^{2}\left[u_{2}^{m-2}-u_{1}^{m-2}\right] \leq f\left(t, u_{2}\right)-f\left(t, u_{1}\right), u_{2}>u_{1}>0 .
$$

Example 1 Obviously this condition is fulfilled for an arbitrary nondecreasing Hölder continuous $f$ if $m \in[1,2]$ and for such $f, m$ above Theorem 1 guarantees the existence of a global strong solution of problem (1.7), (1.2). Note that we can take here $f \equiv 0$ as well.

Example 2 Consider equations

$$
\begin{aligned}
& u_{t}=\left(u^{5 / 2}\right)_{x x}-\lambda u^{1 / 2}, \quad \lambda \geq 15 / 4 K^{2}, \\
& u_{t}=\left(u^{5 / 2}\right)_{x x}-\lambda u^{1 / 4}, \quad \lambda \geq 15 / 2 K^{2} \sqrt{M},
\end{aligned}
$$

where $M=\max _{\Pi_{T}} u=\max _{\mathbf{R}} u_{0}$. One can easily see that (1.4)-(1.6) are fulfilled in both cases, and thus Theorem 1 guarantees the global solvability of problem (1.9), (1.2) and (1.10), (1.2). See also Example 3 in Sect. 2.4.

What happens if no absorption term is present? We will give an answer on this question for the porous medium equation:

$$
u_{t}=\left(u^{m}\right)_{x x} \text { in } \Pi_{T} .
$$

Let us remind (see [2,6]) that in the one-dimensional case the optimal regularity of a weak solution of the Cauchy problem (1.11), (1.2) (with $m>1$ ) is the following one: if $u_{0}^{m-1}$ is 
Lipschitz continuous then $u \in \mathcal{C}^{\beta}$ where $\beta=\min \left\{1,(m-1)^{-1}\right\}$. In particular $\beta=1$ for $m \in(1,2]$ which is consistent with the above Theorem 1 (see Example 1 with $f \equiv 0$ ). In the multidimensional case the situation is more complicated and is not completely set yet (see, for example $[3,16]$ and the references therein).

Recall that by weak solution of problem (1.11), (1.2) we understand (see for example [1, 16]) a nonnegative bounded function $u$, with bounded generalized derivative $\left(u^{m}\right)_{x}$, satisfying the identity

$$
\int_{\Pi_{T}}-u \psi_{t} \mathrm{~d} t \mathrm{~d} x+\int_{\Pi_{T}}\left(u^{m}\right)_{x} \psi_{x} \mathrm{~d} t \mathrm{~d} x=\int_{R} u_{0}(x) \psi(0, x) \mathrm{d} x,
$$

for all smooth $\psi(t, x)$ vanishing for large $|x|$ and for $t=T$. Such solution exists for arbitrary $T>0$, see for example $[1,8,11,16]$.

Theorem 2 (local existence) Assume that condition (1.3) is fulfilled and $m \geq 3$, then for some $T^{*}>0$ there exists a strong solution $u(t, x)$ of problem (1.11), (1.2). Moreover

$$
0 \leq u \leq M \forall(t, x) \in \Pi_{T^{*}}, \quad\left\|u_{x}(t, x)\right\|_{L^{\infty}\left(\Pi_{T^{*}}\right)} \leq K^{*},
$$

and

$$
\|u\|_{L_{2}(\mathbf{R})}^{2}+2\left\|\sqrt{\kappa} u_{x}\right\|_{L_{2}\left(\Pi_{T^{*}}\right)}^{2} \leq\left\|u_{0}\right\|_{L_{2}(\mathbf{R})}^{2} \quad \forall t \in\left(0, T^{*}\right)
$$

where $\Pi_{T^{*}}=\left(0, T^{*}\right) \times \mathbf{R}, T^{*}$ depends only on $m, K^{2}, M^{m-3}$ and $K^{*}$ depends only on $K, T^{*}$.

Actually Theorem 2 states that a weak solution (which as it was mentioned above exists for any $T>0)$ has better differential properties for $t \in\left(0, T^{*}\right)$. Note that $T^{*}$ and $K^{*}$ can be find explicitly.

Thus we give an answer to the question formulated above for $m \in(1,2] \cup[3, \infty)$. For $m \in(2,3)$ our approach seems not working.

Let us turn now to the Dirichlet problem. Consider equation

$$
u_{t}=\left(\kappa(t, u) u_{x}\right)_{x}-f(t, u), \quad(t, x) \in Q_{T}=(0, T) \times(-l, l),
$$

coupled with initial and boundary conditions

$$
u(0, x)=u_{0}(x) \text { for }|x|<l, u_{0}( \pm l)=0 \text { and } u(t, \pm l)=0 \text { for } t \in[0, T] .
$$

We suppose that

$$
0 \leq u_{0}(x) \leq M, \quad\left|u_{0}(x)-u_{0}(y)\right| \leq K|x-y|, \quad \forall x, y \in[-l, l] .
$$

Assume that conditions (1.4), (1.5) are fulfilled and instead of (1.6) we impose the more restrictive one

$$
K^{2}\left[\kappa_{u}\left(t, u_{2}\right)-\kappa_{u}\left(t, u_{1}\right)\right] \leq f\left(t, u_{2}\right)-f\left(t, u_{1}\right) \quad \text { for } u_{2}>u_{1} \geq 0, \quad t \in[0, T]
$$

Note that (1.15) unlike to (1.6) implies that

$$
K^{2} \kappa_{u}(t, u) \leq f(t, u) \text { for } u \geq 0, \quad t \in[0, T]
$$

which will be used below in the boundary gradient estimates (see Lemma 2.2). 
Definition 2 We say that a nonnegative Hölder continuous function $u(t, x)$ is a strong solution of problem (1.12), (1.13) if $u_{x} \in L^{\infty}\left(Q_{T}\right), u_{t} \in L^{2}\left(Q_{T}\right)$ and for an arbitrary smooth $\phi(t, x)$ the following identity is satisfied

$$
\int_{Q_{T}} u_{t} \phi \mathrm{d} t \mathrm{~d} x+\int_{Q_{T}} \kappa(t, u) u_{x} \phi_{x} \mathrm{~d} t \mathrm{~d} x+\int_{Q_{T}} f(t, u) \phi \mathrm{d} t \mathrm{~d} x=0 .
$$

Theorem 3 (global existence) Assume that conditions (1.4), (1.5), (1.14), (1.15) are fulfilled, then for any $T>0$ there exists a strong solution $u(t, x)$ of problem (1.12), (1.13). Moreover

$$
0 \leq u \leq M \quad \forall(t, x) \in Q_{T}, \quad\left\|u_{x}(t, x)\right\|_{L^{\infty}\left(Q_{T}\right)} \leq K .
$$

Example 2 (but not Example 1) can be extended to the Dirichlet problem by obvious way (see also Example 3 in Sect. 2.4).

Let us consider the Dirichlet problem for the standard porous medium equation with $m \geq 3$

$$
u_{t}=\left(u^{m}\right)_{x x}, \quad m \geq 3 \text { in } Q_{T},
$$

coupled with conditions (1.13).

Theorem 4 (local existence) Assume that condition (1.14) is fulfilled, then for some $T^{*}>0$ there exists a strong solution $u(t, x)$ of problem (1.16), (1.13). Moreover

$$
0 \leq u \leq M \quad \forall(t, x) \in Q_{T^{*}}, \quad\left\|u_{x}(t, x)\right\|_{L^{\infty}\left(Q_{T^{*}}\right)} \leq K^{*}
$$

where $Q_{T^{*}}=\left(0, T^{*}\right) \times(-l, l), T^{*}$ depends only on $m, K^{2}, M^{m-3}$ and $K^{*}$ depends only on $K, T^{*}$.

As in Theorem 2, constants $T^{*}$ and $K^{*}$ will be find explicitly (see proof of this theorem in Sect. 2).

Note that the regularized effect of the low order term for the Burgers equation was demonstrated in [14] and was based on the modification of conditions (0.10)-(0.11) from [13]. In the present paper we actually apply similar approach giving another modification of conditions (0.10)-(0.11) from [13].

Different aspects of degenerate equations with absorption were investigated in [4, 7, 12, 15].

The paper is organized as follows. We start with the Dirichlet problem in Sect. 2 and in Sect. 3 we consider the Cauchy problem.

\section{The Dirichlet problem}

\subsection{Regularization}

Rewrite Eq. (1.12) in the nondivergent form and consider the regularized equation

$$
u_{t}=(\kappa(t, u)+\varepsilon) u_{x x}+\kappa_{u}(t, u) u_{x}^{2}-f(t, u),
$$

where $\varepsilon$ is an arbitrary positive constant.

First consider the auxiliary equation

$$
u_{t}=(\bar{\kappa}(t, u)+\varepsilon) u_{x x}+\kappa_{u}(t, u) u_{x}^{2}-\bar{f}(t, u)
$$


coupled with initial and boundary conditions (1.13), here

$$
\bar{\kappa}(t, z)=\left\{\begin{array}{ll}
\kappa(t, z), & \text { for } z \geq 0 \\
0, & \text { for } z<0
\end{array} \quad \bar{f}(t, z)=\left\{\begin{array}{ll}
f(t, z), & \text { for } z \geq 0 \\
0, & \text { for } z<0
\end{array} .\right.\right.
$$

The existence of classical solution $u_{\varepsilon}$ of problem (2.2), (1.13) under the smoothness assumptions (1.4) follows from [13].

\subsection{A priori estimates}

For simplicity in the proofs of lemmas below we omit the subindex $\varepsilon$.

Lemma 2.1 For a classical solution $u_{\varepsilon}(t, x)$ of problem (2.2), (1.11) the estimate

$$
0 \leq u_{\varepsilon}(t, x) \leq M
$$

holds in $Q_{T}$.

Proof For $v=u e^{-\delta t}, \delta>0$ constant, we have

$$
v_{t}-(\bar{\kappa}(t, u)+\varepsilon) v_{x x}-\kappa_{u}(t, u) v_{x}^{2} e^{\delta t}+\delta v=-\bar{f}(t, u) e^{-\delta t} .
$$

Denote by $\Gamma_{T}$ the parabolic boundary of $Q_{T}$, i.e.

$$
\Gamma_{T}=\partial Q_{T} \backslash\{t=T,|x|<l\} .
$$

Suppose that the function $v$ attains its negative minimum at the point $N \in \overline{Q_{T}} \backslash \Gamma_{T}$, then at this point the left-hand side of (2.3) is strictly negative and the right-hand side is zero (since $v<0$ implies $u<0$ implies $\bar{f}(t, u)=0$ ). From this contradiction we conclude that $v$ cannot attain its negative minimum at the internal points and hence, taking into account the initial and the boundary conditions, we conclude that $v(t, x) \geq 0$.

Assume now that the function $v$ attains its positive maximum at the point $N_{1} \in \bar{Q}_{T} \backslash \Gamma_{T}$, then at this point the left-hand side of (2.3) is strictly positive and the right-hand side is nonpositive (since $v>0$ implies $u>0$ implies $\bar{f}(t, u) \geq 0$ ). Hence $v$ cannot attain its positive maximum at the internal points and taking into account the boundary conditions we conclude that $v(t, x) \leq M$ and thus

$$
0 \leq u(t, x) \leq e^{\delta t} M .
$$

Passing to the limit as $\delta \rightarrow 0$ we obtain the needed estimate.

From the above estimate it immediately follows that Eq. (2.2) coincides with Eq. (2.1), so, instead of problem (2.2), (1.13) we will consider problem (2.1), (1.13).

Lemma 2.2 For a classical solution $u_{\varepsilon}(t, x)$ of problem $(2.1),(1.13)$ the estimates

$$
u_{\varepsilon}(t, x) \leq K(x+l), \quad u_{\varepsilon}(t, x) \leq K(l-x)
$$

hold in $Q_{T}$.

Proof Let

$$
v(t, x)=(u(t, x)-K(x+l)) e^{-\delta t} .
$$

By direct calculations we obtain that

$$
v_{t}+\delta v-(\kappa(t, u)+\varepsilon) v_{x x}=e^{-\delta t}\left[\kappa_{u}(t, u) u_{x}^{2}-f(t, u)\right] .
$$


Suppose that at the point $N \in \bar{Q}_{T} \backslash \Gamma_{T}$ the function $v$ attains its positive maximum, we have

$$
v_{t}+\delta v-\left.(\kappa(t, u)+\varepsilon) v_{x x}\right|_{N}>0,
$$

on the other hand at this point

$$
v>0, \quad v_{x}=0 \Leftrightarrow u>0, \quad u_{x}=K
$$

and hence, due to (1.5), (1.15)

$$
\left.e^{-\delta t}\left[\kappa_{u}(t, u) u_{x}^{2}-f(t, u)\right]\right|_{N}=\left.e^{-\delta t}\left[\kappa_{u}(t, u) K^{2}-f(t, u)\right]\right|_{N} \leq 0 .
$$

This contradicts (2.5); thus, $v$ cannot attain its positive maximum at the internal points of $Q_{T}$. Consider $v$ on the parabolic boundary of $Q_{T}$ :

for $t=0$ we have

$$
v(0, x)=\left(u_{0}(x)-K(x+l)\right) e^{-\delta t} \leq 0,
$$

for $x= \pm l$ we have

$$
v(t, \pm l)=(-K( \pm l+l)) e^{-\delta t} \leq 0 .
$$

Hence

$$
v(t, x) \leq 0 \text { for }(t, x) \in \bar{Q}_{T}
$$

and consequently

$$
u(t, x) \leq K(x+l) \text { for }(t, x) \in \bar{Q}_{T} .
$$

Similarly, for

$$
w(t, x)=(u(t, x)-K(l-x)) e^{-\delta t}
$$

we obtain that

$$
w_{t}+\delta w-(\kappa(t, u)+\varepsilon) w_{x x}=e^{-\delta t}\left[\kappa_{u}(t, u) u_{x}^{2}-f(t, u)\right]
$$

Suppose that at the point $N_{1} \in \bar{Q}_{T} \backslash \Gamma_{T}$ the function $w$ attains its positive maximum, then at this point the left-hand side of (2.6) is strictly positive, on the other hand at this point

$$
u>0, \quad u_{x}=-K
$$

and hence, due to (1.5), (1.15) the right-hand side of (2.6) is less or equal zero. From this contradiction we conclude that $w$ cannot attain its positive maximum at the internal points of $Q_{T}$. Consider $w$ on the parabolic boundary of $Q_{T}$ :

$$
w(0, x)=\left(u_{0}(x)-K(l-x)\right) e^{-\delta t} \leq 0, w(t, \pm l)=(-K(l \mp l)) e^{-\delta t} \leq 0 .
$$

Hence

$$
w(t, x) \leq 0 \text { for }(t, x) \in \bar{Q}_{T}
$$

and consequently

$$
u(t, x) \leq K(l-x) \text { for }(t, x) \in \bar{Q}_{T} .
$$


Lemma 2.3 For a classical solution of problem (2.1), (1.13) the estimate

$$
\left|u_{\varepsilon x}(t, x)\right| \leq K
$$

holds in $Q_{T}$.

Proof Consider Eq. (2.1) at two different points $(t, x),(t, y), x>y$ :

$$
\begin{array}{ll}
u_{t}=(\kappa(t, u)+\varepsilon) u_{x x}+\kappa_{u}(t, u) u_{x}^{2}-f(t, u), & u=u(t, x), \\
u_{t}=(\kappa(t, u)+\varepsilon) u_{y y}+\kappa_{u}(t, u) u_{y}^{2}-f(t, u), & u=u(t, y) .
\end{array}
$$

Subtracting (2.8) from (2.7) for

$$
\bar{w}(t, x, y)=u(t, x)-u(t, y)-K(x-y)
$$

we obtain

$$
\begin{aligned}
& \bar{w}_{t}-(\kappa(t, u(t, x))+\varepsilon) \bar{w}_{x x}-(\kappa(t, u(t, y))+\varepsilon) \bar{w}_{y y} \\
& =\kappa_{u}(t, u(t, x)) u_{x}^{2}(t, x)-f(t, u(t, x))-\kappa_{u}(t, u(t, y)) u_{y}^{2}(t, y)+f(t, u(t, y))
\end{aligned}
$$

and for $w=\bar{w} e^{-t}$ we have

$$
\begin{aligned}
L w \equiv & w_{t}+w-(\kappa(t, u(t, x))+\varepsilon) w_{x x}-(\kappa(t, u(t, y))+\varepsilon) w_{y y} \\
= & {\left[\kappa_{u}(t, u(t, x)) u_{x}^{2}(t, x)-f(t, u(t, x))\right.} \\
& \left.-\kappa_{u}(t, u(t, y)) u_{y}^{2}(t, y)+f(t, u(t, y))\right] e^{-t} .
\end{aligned}
$$

Consider Eq. (2.9) in the domain

$$
P=\{(t, x, y): t \in(0, T), x \in(-l, l), y \in(-l, l), x>y\} .
$$

Denote by $\Gamma$ the parabolic boundary of $P$, i.e.

$$
\Gamma=\partial P \backslash\{t=T, x \in(-l, l), y \in(-l, l), x>y\} .
$$

Suppose that the function $w$ attains its positive maximum at the point $N \in \bar{P} \backslash \Gamma$, then at this point we have

$$
u(t, x)>u(t, y) \text { and } u_{x}(t, x)=u_{y}(t, y)=K,
$$

hence due to condition (1.5)

$$
\left.L w\right|_{N}<0
$$

which is impossible. Consequently $w$ cannot attain its positive maximum at the internal points of $P$. Consider the parabolic boundary of the domain $P$ :

1. for $t=0$ we have $w(0, x, y)=u_{0}(x)-u_{0}(y)-K(x-y) \leq 0$;

2. for $x=y$ we have $w=0$;

3. for $x=l, y \in[-l, l]$, since $-u(t, y) \leq 0$, we have

$$
w(t, l, y)=(-u(t, y)-K(l-y)) e^{-t} \leq 0 ;
$$

and finally

4. for $y=-l, x \in[-l, l]$, due to Lemma 2.2, we have

$$
w(t, x,-l)=(u(t, x)-K(x+l)) e^{-t} \leq 0 .
$$


Thus we obtain that for $x>y$

$$
u(t, x)-u(t, y) \leq K(x-y) .
$$

Similarly, subtracting (2.7) from (2.8) for

$$
v(t, x, y)=(u(t, y)-u(t, x)-K(x-y)) e^{-t}
$$

we obtain

$$
\begin{aligned}
L v= & {\left[\kappa_{u}(t, u(t, y)) u_{y}^{2}(t, y)-f(t, u(t, y))\right.} \\
& \left.-\kappa_{u}(t, u(t, x)) u_{x}^{2}(t, x)+f(t, u(t, x))\right] e^{-t} .
\end{aligned}
$$

Consider Eq. (2.10) in the domain $P$. Suppose that the function $v$ attains its positive maximum at the point $N \in \bar{P} \backslash \Gamma$, then at this point we have

$$
u(t, y)>u(t, x) \text { and } u_{y}(t, y)=u_{x}(t, x)=-K,
$$

hence due to condition (1.5)

$$
\left.L v\right|_{N}<0
$$

which is impossible. Thus $v$ cannot attain its positive maximum at the internal points of $P$. Consider the parabolic boundary of the domain $P$ :

1. for $t=0$ we have $v(0, x, y)=u_{0}(y)-u_{0}(x)-K(x-y) \leq 0$;

2. for $x=y$ we have $v=0$;

3. for $x=l, y \in[-l, l]$ due to Lemma 2.2, we have

$$
v(t, l, y)=(u(t, y)-K(l-y)) e^{-t} \leq 0,
$$

and finally

4. for $y=-l, x \in[-l, l]$ we have

$$
v(t, x,-l)=(-u(t, x)-K(x+l)) e^{-t} \leq 0
$$

since $-u(t, y) \leq 0$.

Thus we obtain that for $x>y$

$$
|u(t, x)-u(t, y)| \leq K(x-y) .
$$

In view of the symmetry of the variables $x, y$ we conclude that for $t \in[0, T],|x| \leq l$, $|y| \leq l$ we have

$$
|u(t, x)-u(t, y)| \leq K|x-y|
$$

which implies the required estimate.

Lemma 2.4 For a classical solution of problem (2.1), (1.13) the estimate

$$
\int_{Q_{T}} u_{\varepsilon t}^{2}(t, x) \mathrm{d} t \mathrm{~d} x \leq C
$$

holds, where the constant $C$ do not depend on $\varepsilon$. 
Proof Rewrite Eq. (2.1) in divergent form

$$
\left.u_{\varepsilon t}=\left(\kappa\left(t, u_{\varepsilon}\right)+\varepsilon\right) u_{\varepsilon x}\right)_{x}-f\left(t, u_{\varepsilon}\right) .
$$

Multiplying (2.11) by $u_{t}$ and integrating by parts with respect to $x$ we obtain

$$
\int_{-l}^{l} u_{t}^{2} \mathrm{~d} x=-\frac{1}{2} \int_{-l}^{l}(\kappa+\varepsilon)\left(u_{x}^{2}\right)_{t} \mathrm{~d} x-\int_{-l}^{l} f u_{t} \mathrm{~d} x .
$$

Now integrating by parts with respect to $t$ we obtain

$$
\begin{aligned}
\int_{Q_{T}} u_{t}^{2} \mathrm{~d} t \mathrm{~d} x= & -\left.\frac{1}{2} \int_{-l}^{l}(\kappa+\varepsilon) u_{x}^{2}\right|_{t=0} ^{t=T} \mathrm{~d} x+\frac{1}{2} \int_{Q_{T}} \kappa_{t} u_{x}^{2} \mathrm{~d} t \mathrm{~d} x \\
& +\frac{1}{2} \int_{Q_{T}} \kappa_{u} u_{t} u_{x}^{2} \mathrm{~d} t \mathrm{~d} x-\int_{Q_{T}} f u_{t} \mathrm{~d} t \mathrm{~d} x .
\end{aligned}
$$

Applying the Hölder and Young inequalities we conclude

$$
\begin{aligned}
& \left.\int_{Q_{T}} u_{t}^{2} \mathrm{~d} t \mathrm{~d} x+2 \int_{-l}^{l}(\kappa(T, u(T, x))+\varepsilon) u_{x}^{2}(T, x) \mathrm{d} x\right) \\
& \leq 2 \int_{-l}^{l}\left(\kappa\left(0, u_{0}(x)\right)+\varepsilon\right) u_{0}^{2}(x) \mathrm{d} x+2 \int_{Q_{T}}\left|\kappa_{t}\right| u_{x}^{2} \mathrm{~d} t \mathrm{~d} x \\
& \quad+\int_{Q_{T}} \kappa_{u} u_{x}^{4} \mathrm{~d} t \mathrm{~d} x+2 \int_{Q_{T}} f^{2} \mathrm{~d} t \mathrm{~d} x,
\end{aligned}
$$

from where the assertion of the Lemma follows.

\subsection{Proof of Theorem 3}

We have constructed functions $u_{\varepsilon}$-classical solutions of (2.1), (1.2). Multiplying (2.11) by an arbitrary smooth $\phi$ and integrating by part we obtain

$$
\begin{aligned}
\int_{Q_{T}} & u_{\varepsilon t} \phi \mathrm{d} t \mathrm{~d} x+\int_{Q_{T}}\left(\kappa\left(t, u_{\varepsilon}\right)+\varepsilon\right) u_{\varepsilon x} \phi_{x} \mathrm{~d} t \mathrm{~d} x+\int_{Q_{T}} f\left(t, u_{\varepsilon}\right) \phi \mathrm{d} t \mathrm{~d} x \\
= & \left.\varepsilon \int_{0}^{T} u_{\varepsilon x} \phi\right|_{x=-l} ^{x=l} \mathrm{~d} t .
\end{aligned}
$$

Note that the for $u_{\varepsilon}$ the following estimate (see $[5,9]$ ) takes place:

$$
\left|u_{\varepsilon}\left(t_{1}, x\right)-u_{\varepsilon}\left(t_{2}, x\right)\right| \leq C\left|t_{1}-t_{2}\right|^{1 / 2}
$$

with constant $C$ depending only on $M, K$ and $\max (\kappa+\varepsilon)$, $\max \kappa_{u}$, max $f$ (maximum is taken over the set $[0, T] \times[0, M])$. The above estimate and Lemmas 2.1, 2.3, 2.4 guarantee the existence of subsequence $\varepsilon_{n}$ such that

$$
\begin{aligned}
& u_{\varepsilon_{n}} \rightarrow u \text { uniformly, } \\
& u_{\varepsilon_{n} x} \rightarrow u_{x}{ }^{*} \text { weakly in } L_{\infty}\left(Q_{T}\right), \\
& u_{\varepsilon_{n} t} \rightarrow u_{t}{ }^{*} \text { weakly in } L_{2}\left(Q_{T}\right)
\end{aligned}
$$

as $n \rightarrow \infty\left(\varepsilon_{n} \rightarrow 0\right)$. Based on these we can pass to the limit in the identity (2.12) and by this prove the existence. 


\subsection{Proof of Theorem 4}

For the function $v=u e^{-\mu t}, \mu>0$ the problem (1.16), (1.13) takes the form

$$
\begin{aligned}
& v_{t}=\left(\kappa(t, v) v_{x}\right)_{x}-\mu v, \quad m \geq 3, \\
& v(0, x)=u_{0}(x) \geq 0, \quad v(t, \pm l)=0,
\end{aligned}
$$

with

$$
\kappa(t, v)=m e^{\mu(m-1) t} v^{m-1} .
$$

Condition (1.15) in this case can be written as follows

$$
m(m-1) K^{2}\left[v_{2}^{m-2}-v_{1}^{m-2}\right] \leq \mu e^{\mu(1-m) t}\left[v_{2}-v_{1}\right], \quad v_{2}>v_{1} \geq 0
$$

or

$$
t \leq \frac{1}{\mu(m-1)} \ln \left(\frac{\mu}{m(m-1) K^{2}} \frac{v_{2}-v_{1}}{v_{2}^{m-2}-v_{1}^{m-2}}\right) .
$$

The last is fulfilled if

$$
t \leq \frac{1}{\mu(m-1)} \ln \left(\frac{\mu}{m(m-1) K^{2}} \frac{1}{(m-2) M^{m-3}}\right),
$$

where $M=\max v=\max u$. Define $T^{*}>0$ by the following

$$
T^{*}=\sup _{\mu} \frac{1}{\mu(m-1)} \ln \left(\frac{\mu}{m(m-1)(m-2) K^{2} M^{m-3}}\right),
$$

here supremum is taking for $\mu>m(m-1)(m-2) K^{2} M^{m-3}$. Thus Theorem 3 guarantees the existence of a strong solution of problem (2.14), (2.15) on the interval $\left(0, T^{*}\right)$ and the estimate

$$
\left\|v_{x}\right\|_{L^{\infty}\left(0, T^{*}\right)} \leq K=\max \left|v_{x}(0, x)\right|=\max \left|u_{0 x}(x)\right| .
$$

Returning to problem (1.16), (1.13) we finish the proof of the existence. Obviously

$$
\left\|u_{x}\right\|_{L^{\infty}\left(0, T^{*}\right)} \leq K e^{\mu t} \leq K^{*}=K e^{\mu T^{*}} .
$$

Example 3 Consider equation

$$
u_{t}=\left(u^{3}\right)_{x x}-\lambda u, \quad \lambda \geq 6 K^{2} \text { in } Q_{T},
$$

One can easily see that (1.15) is fulfilled and thus Theorem 3 guarantees the global solvability of problem (2.17), (1.13).

Now consider equation

$$
u_{t}=\left(u^{3}\right)_{x x} \text { in } Q_{T},
$$

For $v=u e^{-\mu t}$ we obtain

$$
v_{t}=\left(3 e^{2 \mu t} v^{2} v_{x}\right)_{x}-\mu t .
$$

Condition (2.16) takes the form

$$
6 K^{2} \leq \mu e^{-2 \mu t} \Leftrightarrow t \leq \frac{1}{2 \mu} \ln \frac{\mu}{6 K^{2}} .
$$


Supremum of $1 / 2 \mu \ln \left(\mu / 6 K^{2}\right)$ (with respect to $\mu$ under restriction $\mu>6 K^{2}$ ) is obtained for $\mu=e 6 K^{2}$, hence for

$$
t \in\left(0, \frac{1}{12 e K^{2}}\right)
$$

we have

$$
\left|v_{x}(t, x)\right| \leq K \Leftrightarrow\left|u_{x}(t, x)\right| \leq K e^{6 e K^{2} t} \leq K^{*}=K \sqrt{e} .
$$

Thus we conclude that Theorem 4 guarantees the existence of a strong solution to the problem (2.18), (1.13) on the interval

$$
t \in\left(0, T^{*}\right), \quad T^{*}=\frac{1}{12 e K^{2}} .
$$

\section{The Cauchy problem}

\subsection{Regularized problem}

Consider the following regularized problem

$$
u_{t}=(\kappa(t, u)+\varepsilon) u_{x x}+\kappa_{u}(t, u) u_{x}^{2}-f(t, u) \text { in } Q_{T}^{l}=(0, T) \times(-l, l)
$$

coupled with initial and boundary conditions

$$
u(0, x)=h_{l}(x), \quad u_{x}(t, \pm l) \pm \delta u(t, \pm l)=0,
$$

where

$$
0 \leq h_{l}(x) \leq M, \quad\left|h_{l}^{\prime}(x)\right| \leq K, \quad \forall x \in[-l, l]
$$

and $h_{l x}( \pm l) \pm \delta h_{0}( \pm l)=0$, here $\varepsilon$ and $l$ are arbitrary positive constants and positive constant $\delta$ is such that

$$
M \delta<K
$$

(we will use the last inequality in the proof of Lemma 3.2).

First consider the following auxiliary equation

$$
u_{t}=(\bar{\kappa}(t, u)+\varepsilon) u_{x x}+\kappa_{u}(t, u) u_{x}^{2}-\bar{f}(t, u) \text { in } Q_{T}^{l}
$$

where functions $\bar{\kappa}(t, u), \bar{f}(t, u)$ where defined in the previous section.

The existence of classical solution of problem (3.3), (3.2) under the smoothness assumptions (1.4) follows from [13].

\subsection{A priori estimates}

Lemma 3.1 For a classical solution $u_{\varepsilon}(t, x)$ of problem (3.3), (3.2) the estimate

$$
0 \leq u_{\varepsilon}(t, x) \leq M
$$

holds in $Q_{T}$. 
Proof The proof is similar to the proof of Lemma 2.1. The only difference is on the lateral boundary of the domain, i.e. $x= \pm l, t \in[0, T]$ where for $v=u e^{-\delta t}$ we have

$$
v_{x}(t, \pm l) \pm \delta v(t, \pm l)=0 .
$$

Hence on the lateral boundary the function $v$ cannot attain positive maximum or negative minimum. Thus, similarly to the proof of Lemma 2.1 we conclude that the function $v$ attains its positive maximum and negative minimum at the initial moment and consequently

$$
0 \leq u \leq M .
$$

From the above estimate it immediately follows that equation (3.3) coincides with equation (3.1), so, instead of problem (3.3), (3.2) we will consider problem (3.1), (3.2).

Lemma 3.2 For a classical solution of problem (3.1), (3.2) the estimate

$$
\left|u_{\varepsilon x}(t, x)\right| \leq K
$$

holds in $Q_{T}$.

Proof The proof is similar to the proof of Lemma 2.3. The only difference is on the lateral boundary of the domain $P$.

Consider the function $w(t, x, y)$ (see the proof of Lemma 2.3) on the lateral boundary of the domain $P$ :

1. for $x=y$ we have $w=0$;

2. for $x=l, y \in[-l, l]$ we have

$$
w_{x}(t, l, y)=\left(u_{x}(t, l)-K\right) e^{-t}=(-\delta u(t, l)-K) e^{-t}<0
$$

and thus the function $w$ cannot attain its positive maximum on this part of the lateral boundary;

3. for $y=-l, x \in[-l, l]$ we have

$$
w_{y}(t, x,-l)=\left(-u_{y}(t,-l)+K\right) e^{-t}=(-\delta u(t,-l)+K) e^{-t}>0
$$

and the function $w$ cannot attain its positive maximum on this part of the lateral boundary as well (recall that $K>\delta M$ and note that on this part of the boundary the derivative with respect to $y$ is inward derivative).

Thus similarly to Lemma 2.3 we conclude that for $x>y$

$$
u(t, x)-u(t, y) \leq K(x-y) .
$$

Now consider the function $v(t, x, y)$ on the lateral boundary of the domain $P$ :

1. for $x=y$ we have $v=0$;

2. for $x=l, y \in[-l, l]$ we have

$$
v_{x}(t, l, y)=\left(-u_{x}(t, l)-K\right) e^{-t}=(\delta u(t, l)-K)<0,
$$

3. for $y=-l, x \in[-l, l]$ we have

$$
v_{y}(t, x,-l)=\left(u_{y}(t,-l)+K\right) e^{-t}=(\delta u(t,-l)+K) e^{-t}>0
$$

and hence the function $v$ cannot attain its positive maximum on part 2. and 3. of the lateral boundary. 
Thus we obtain that for $x>y$

$$
|u(t, x)-u(t, y)| \leq K(x-y) .
$$

In view of the symmetry of the variables $x y$ we conclude that for $t \in[0, T],|x| \leq l$, $|y| \leq l$ we have

$$
|u(t, x)-u(t, y)| \leq K|x-y|
$$

which implies the required estimate.

\subsection{Proof of Theorems 1 and 2}

We have constructed functions $u_{\varepsilon}$-classical solutions of (3.1), (3.2). Note that the estimates of $u_{\varepsilon}, u_{\varepsilon x}$ as well as the estimate

$$
\left|u_{\varepsilon}\left(t_{1}, x\right)-u_{\varepsilon}\left(t_{2}, x\right)\right| \leq C\left|t_{1}-t_{2}\right|^{1 / 2}
$$

are independent of $\delta$ (and of $\varepsilon$ ). From Schauder estimates we conclude that for some $\gamma \in(0,1)$ the $\mathcal{C}_{t, x}^{1+\gamma / 2,2+\gamma}$ norm of $u_{\varepsilon}$ is bounded by a constant independent of $\delta$ (but of course depending on $\varepsilon$ ). Thus we can pass to the limit as $\delta \rightarrow 0$ and obtain the classical solution of the homogeneous Neumann problem:

$$
\begin{aligned}
& u_{\varepsilon t}=\left(\kappa\left(t, u_{\varepsilon}\right)+\varepsilon\right) u_{\varepsilon x x}+\kappa_{u}\left(t, u_{\varepsilon}\right) u_{\varepsilon x}^{2}-f\left(t, u_{\varepsilon}\right) \text { in } Q_{T}^{l}, \\
& u(0, x)=h_{l}(x), \quad u_{x}(t, \pm l)=0,
\end{aligned}
$$

where $h_{l x}( \pm l)=0$.

Obviously the estimates of Lemma 3.1 and 3.2 as well as the estimate (3.4) hold for the solution of problem (3.5), (3.6). Let us obtain the needed integral estimates.

Lemma 3.3 For a classical solution of problem (3.5), (3.6) the estimate

$$
\int_{Q_{T}} u_{\varepsilon t}^{2} \mathrm{~d} t \mathrm{~d} x \leq C
$$

holds, where the constant $C$ do not depend on $\varepsilon$.

The proof is similar to the proof of Lemma 2.4.

Lemma 3.4 For a classical solution of problem (3.5), (3.6) the estimate

$$
\int_{-l}^{l} u_{\varepsilon}^{2} \mathrm{~d} x+2 \int_{Q_{T}} \kappa\left(t, u_{\varepsilon}\right) u_{\varepsilon x}^{2} \mathrm{~d} x \mathrm{~d} t \leq \int_{-l}^{l} u_{0}^{2}(x) \mathrm{d} x
$$

holds $\forall t \in(0, T)$, where the constant $C$ do not depend on $\varepsilon$.

Proof Multiplying (3.5) by $u$ and integrating by parts we obtain

$$
\frac{1}{2} \frac{\mathrm{d}}{\mathrm{d} t} \int_{-l}^{l} u^{2} \mathrm{~d} x+\int_{-l}^{l}(\kappa(t, u)+\varepsilon) u_{x}^{2} \mathrm{~d} x=-\int_{-l}^{l} f u \mathrm{~d} x \leq 0 .
$$

Integrating with respect to $t$ we obtain the required estimate.

Let us extend $h_{l}(x)$ by zero for $|x|>l$. Now we approach the initial function $u_{0}$ by (extended) functions $h_{l}$ and obtain the (classical) solution of the Cauchy problem

$$
u_{\varepsilon t}=\left(\kappa\left(t, u_{\varepsilon}\right)+\varepsilon\right) u_{\varepsilon x x}+\kappa_{u}\left(t, u_{\varepsilon}\right) u_{\varepsilon x}^{2}-f\left(t, u_{\varepsilon}\right) \text { in } \Pi_{T},
$$




$$
u(0, x)=u_{0}(x)
$$

passing to the limit as $l \rightarrow \infty$ and applying the standard diagonal process ( [10]). Note that the a priori estimates obtained above are independent of $l$ and hence are valid for the problem (3.7), (3.8) as well.

Thus there exists a subsequence $\varepsilon_{n}$ such that

$$
\begin{aligned}
& u_{\varepsilon_{n}} \rightarrow u \text { uniformly, } \\
& u_{\varepsilon_{n} x} \rightarrow u_{x} *_{\text {weakly in }} L_{\infty}\left(\Pi_{T}\right) \text {, } \\
& u_{\varepsilon_{n} t} \rightarrow u_{t}{ }^{*} \text { weakly in } L_{2}\left(\Pi_{T}\right)
\end{aligned}
$$

as $n \rightarrow \infty\left(\varepsilon_{n} \rightarrow 0\right)$. Based on these we can pass to the limit in the identity

$$
\int_{\Pi_{T}} u_{\varepsilon t} \phi \mathrm{d} t \mathrm{~d} x+\int_{\Pi_{T}}\left(\kappa\left(t, u_{\varepsilon}\right)+\varepsilon\right) u_{\varepsilon x} \phi_{x} \mathrm{~d} t \mathrm{~d} x+\int_{\Pi_{T}} f\left(t, u_{\varepsilon}\right) \phi \mathrm{d} t \mathrm{~d} x=0
$$

with an arbitrary smooth $\phi(t, x)$ vanishing for large $|x|$. By this we finish the proof of Theorem 1.

The proof of Theorem 2 is similar to the proof of Theorem 4.

Note that Example 3 by obvious way can be extended to the Cauchy problem.

\section{References}

1. Aronson, D.G.: The porous medium equations. In: Nonlinear Diffusion Problems. Lecture Notes in Mathematics, vol. 1224, pp. 1-46. Springer (1986)

2. Aronson, D.G.: Regularity properties of flows through porous media. SIAM J. Appl. Math. 17, 461-467 (1969)

3. Dibenedetto, E.: Interior and boundary regularity for a class of free boundary problems. In: Free Boundary Problems, Theory and Applications, Vol. II. Proceedings of Interdisciplinary Symposium, Montecatini/Italy (1981). Research Notes in Mathematics, vol. 79, pp. 383-396 (1983)

4. Galaktionov, V., Shmarev, S., Vazquez, J.: Regularity of interfaces in diffusion processes under the influence of strong absorption. Arch. Ration. Mech. Anal. 149(3), 183-212 (1999)

5. Gilding, B.H.: Hölder continuity of solutions of parabolic equations. J. Lond. Math. Soc. 13(1), 103-106 (1976)

6. Gilding, B.H., Peletier, L.A.: The Cauchy problem for an equation in the theory of infiltration. Arch. Ration. Mech. Anal. 16, 127-140 (1976)

7. Jäger, W., Lu, Y.: Global regularity of solution for general degenerate parabolic equations in 1-D. J. Differ. Equ. 140, 365-377 (1997)

8. Kalashnikov, A.S.: The Cauchy problem and boundary problems for equations of the type of non-stationary filtration. Izv. Acad. Nauk SSSR Ser. Mat. 22, 667-704 (1958)

9. Kruzhkov, S.N.: Quasilinear parabolic equations and systems with two independent variables. Trudy Sem. Petrovsk. 5, 217-272 (1979) (Russian). English transl. in: Topics in Modern Math., Consultant Bureau, New York 1985

10. Ladyzhenskaja, O.A., Solonnikov, V.A., Uraltseva, N.A.: Linear and Quasilinear Equations of Parabolic Type. Translations of Mathematical Monographs, vol. 23. Amer. Math. Soc., Providence (1968)

11. Oleinik, O.A., Kalashnikov, A.S., Chzou, Y.-I.: The Cauchy problem and boundary problems for equations of the type of non stationary filtration. Izv. Akad. Nauk SSSR Ser. Math. 22, 667-704 (1958)

12. Shmarev, S.I.: Interfaces in multidimensional diffusion equations with absorption terms. Nonlinear Anal. 53(6), 791-828 (2003)

13. Tersenov, Al, Tersenov, Ar: On the Bernstein-Nagumo's condition in the theory of nonlinear parabolic equations. J. Reine Angew. Math. 572, 197-217 (2004)

14. Tersenov, Al: On the generalized Burgers equation. NoDEA Nonlinear Differ. Equ. Appl. 17, 437-452 (2010)

15. Tersenov, A.: On the fast diffusion with strong absorption. J. Math. Phys. 54(4), 041503 (2013)

16. Vazquez, J.L.: The Porous Medium Equation. Oxford University Press, Oxford (2007) 